\title{
(2) OPEN ACCESS \\ Partial breast resection for multifocal lower quadrant breast tumour using virtual reality
}

\author{
Enora Laas (D) ,' Mohamed El Beheiry (D) ,'3 Jean-Baptiste Masson (D) , 2,3 \\ Caroline Malhaire (i) ${ }^{4}$
}

${ }^{1}$ Surgery Department, Institut Curie, Paris, France

${ }^{2}$ Decision and Bayesian Computation, Neuroscience Department, CNRS UMR 3571, Institut Pasteur, Paris, France ${ }^{3}$ Decision and Bayesian Computation, Computational Biology Department, CNRS USR 3756, Institut Pasteur, Paris, France

${ }^{4}$ Medical Imaging Department, Institut Curie, Paris, France

\section{Correspondence to \\ Mohamed El Beheiry; mohamed.el-beheiry@pasteur. fr and \\ Mr Jean-Baptiste Masson; jean-baptiste.masson@pasteur. fr}

J-BM and CM contributed equally.

EL and MEB contributed equally.

EL and MEB are joint first authors.

J-BM and CM are joint senior authors.

Accepted 26 February 2021

Check for updates

(c) BMJ Publishing Group Limited 2021. Re-use permitted under CC BY-NC. No commercial re-use. See rights and permissions. Published by BMJ.

To cite: Laas E, El Beheiry M, Masson J-B, et al. BMJ Case Rep 2021;14:e241608. doi:10.1136/bcr-2021-

241608

\section{SUMMARY}

Oncoplastic surgery allows an increase in the number of indications for conservative breast cancer treatments. However, uncertainty as to whether it can be performed still exists in certain situations such as with multicentric or multifocal lesions, even when the breast volume can accommodate it. With the aid of a virtual reality software, DIVA, allowing the precise visualisation of tumours and breast volumes based entirely on the patient's MRI, we report the ability to rapidly confirm and secure an indication for partial surgery of multiple lesions in a 31-year-old patient. With the described approach, the patient did not have to suffer significant disfigurement from cancerous breast surgery without compromising safety.

\section{CASE PRESENTATION}

A 31-year-old patient was admitted at the Institut Curie hospital centre in Paris, France after selfpalpating nodules in her right breast. She had no personal or family history of malignancy or significant medical problems. She had stopped breast feeding her 16-month-old child 3 months prior to hospital admission. The patient was $159 \mathrm{~cm}$ tall and weighed $57 \mathrm{~kg}$, with an E cup bra size.

Clinical breast examination revealed a main mass of $50 \mathrm{~mm}$ in the lower inner quadrant of the right breast and a second mass located $25 \mathrm{~mm}$ lower in the submammary fold.

Mammography was classified as American College of Radiology (ACR) category 2 on the left breast and ACR category 5 on the right breast. In the right breast, the mammography showed the presence of four irregular masses: a single bilobed mass in the lower inner quadrant and three masses at the union of the lower quadrants. There was no evidence of calcifications.

On ultrasonography of the right breast, the single bilobed lesion was found in the lower inner quadrant, at 4 o'clock radius $9 \mathrm{~cm}$ from the nipple, and measured $32 \times 26 \times 21 \mathrm{~mm}$. The other tumour masses consisted of three contiguous lesions at the union of the lower quadrants, at 6 o'clock radius $4 \mathrm{~cm}$ from the nipple, extending $45 \times 29 \mathrm{~mm}$ and $36 \mathrm{~mm}$ apart from the inner edge of the single bilobed lesion in the lower inner quadrant. There were no lesions detected in the upper quadrants and the lower outer quadrant. The total area of the lesions extended over $8 \mathrm{~cm}$ wide, $45 \mathrm{~mm}$ high and $6 \mathrm{~cm}$ anteroposterior. A right axillary lymphadenopathy was also present.
Two needle microbiopsies were performed on the two main lesions of the right breast:

- In the lower inner quadrant, the biopsy found an infiltrating ductal carcinoma of grade EE II oestrogen receptor (ER) $0 \%$, progesterone receptor (PR) 0\%, human epidermal growth factor receptor 2 (HER2) score 0 and Ki-67 90\% (histopathology image is shown in figure 1).

- At the union of the inner quadrants, the biopsy found an infiltrating ductal carcinoma of grade EE II ER 0\%, PR 0\%, HER2 score 0 and Ki-67 $60 \%$.

- An axillary fine needle aspiration biopsy was positive for cancerous cells.

An MRI was performed, confirming multifocal (MF) lesions in the right breast consisting of three large necrotic non-circumscribed masses with satellite nodules along the union of the lower quadrants with axillary lymph node involvement.

A positron emission tomography scan was also performed, revealing hypermetabolic lesions of the right lower quadrants: lower inner and lower outer quadrants and ipsilateral right axillary and right internal mammary lymph node involvement. There was no remote visceral or bone damage.

In this context, a neoadjuvant chemotherapy (NAC) was started. A coil was placed in the uppermost lesion as a guide for future surgery.

The patient first received high doses of four cycles of adriamycin $60 \mathrm{mg}$ and cyclophosphamide $600 \mathrm{mg}$

At the intermediate mammographic and ultrasound check-up, a partial response of the right breast masses was observed $(-30 \%$ according to the response evaluation criteria in solid tumours). Axillary and internal mammary lymphadenopathy had normalised.

Chemotherapy was continued with 12 cycles of weekly paclitaxel combined with carboplatin. At the end of chemotherapy, the masses were no longer clinically palpable.

Ultrasonography confirmed the total disappearance of the lower inner quadrant masses, except for one lesion that decreased in size to $21 \times 4 \times 8 \mathrm{~mm}$ compared with $26 \times 11 \times 21 \mathrm{~mm}$ initially.

On T1-weighted contrast-enhanced MRI, the tumour response was partial, with persistence of two foci in place of the lesions initially described in the lower quadrants. Nodes were normalised.

Given the initial presence of MF lesions in the lower quadrants, radical treatment was deemed necessary. However, given the breast volume (E 


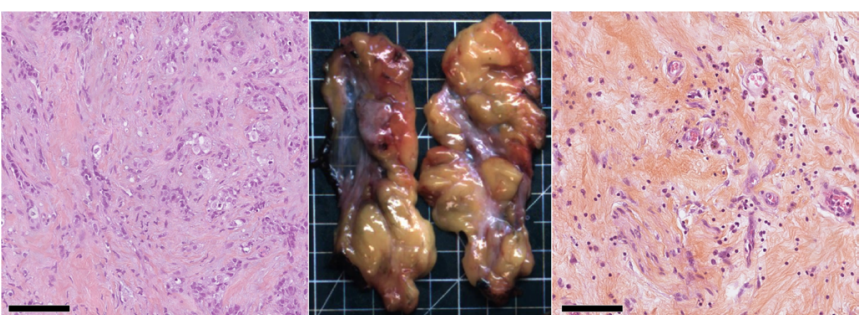

Figure 1 (Left) Breast microbiopsy of a mass of $45 \mathrm{~mm}$ in the lower inner quadrant classed as ACR 5 (histopathological image, hematoxylineosin-safran (HES) colouration): invasive carcinoma of no special type with a trabecular architecture made of tumour cells with moderate cytonuclear atypia within a fibrous stroma with few lymphocytes (black bar corresponds to $100 \mu \mathrm{m}$ ). (Centre) Macroscopic image of the surgical specimen indicating fibrous scarring (grid square corresponds to $1 \mathrm{~cm}$ ). (Right) Surgical specimen/lumpectomy (histopathological image, HES colouration): complete histological response with a dense fibrous reorganisation associated with a polymorphic inflammatory infiltrate linked to the chemotherapy at the site of the identified lesions without residual tumour cells (black bar corresponds to $100 \mu \mathrm{m}$ ). ACR, American College of Radiology.

cup), our team wanted to see if conservative treatment could be considered. Therefore, we decided to use our novel software platform DIVA to guide and limit the surgical excision as possible.

\section{INVESTIGATIONS}

DIVA is a previously reported software platform that allows visualisation and interaction of to-scale patient volumetric representations, or 'avatars', based on the patient's CT or MRI medical image (ie, native Digital Imaging and Communications in Medicine or DICOM image format) in an immersive virtual reality (VR) context. ${ }^{12}$ VR immerses users in an entirely computergenerated environment where three-dimensional (3D) perception is made natural through the integration of stereoscopy and motion tracking.

The DIVA software includes two modes of use. The first is a desktop mode where a volumetric representation of the patient can be visualised and modified to the user's preference in real time using a 3D look-up table, or transfer function, interface. Volumetric patient representations are generated by a volume ray casting approach that maps each voxel to an opacity and colour based on a rendering equation. ${ }^{3}$ This process does not require the medical image to be segmented or pretreated; it simply requires the raw DICOM image extracted from the hospital's Picture Archiving and Communication System.

DIVA's second usage mode makes use of VR to allow the medical practitioner to explore the patient's avatar in an immersive environment conducive to rapidly and intuitively understanding 3D geometries. With the aid of a handheld VR controller, the user can grasp and move the avatar in all directions, cut through the patient from arbitrary angles and fluidly navigate inside the virtual patient (see video 1). A DICOM sampler tool allows original imaging data (here, an MRI) to be simultaneously visualised and related to the volumetric data in VR. Additionally, various annotations may be added in the virtual environment (eg, landmarks and distance measurements). In this study we used the HTC Vive VR headset and its included controller.

Using DIVA and T1-weighted contrast-enhanced MRI, the initial lesions could be directly localised and apprehended in relation to the global volume of the breast. The adjustment

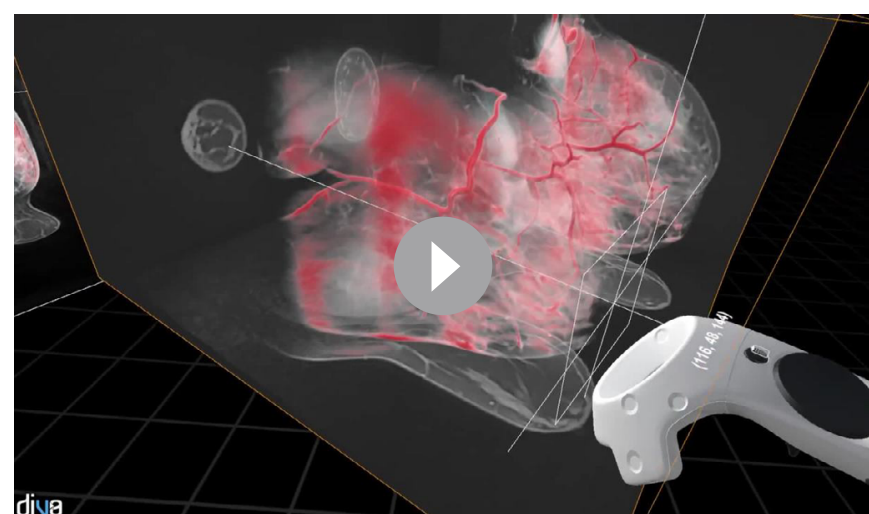

Video 1 Immersive visualisation of to-scale patient avatars based on prechemotherapy and postchemotherapy T1-weighted contrastenhanced MRI using the DIVA software. Using the HTC Vive VR headset, the user navigates inside the patient by orienting and cutting inside the volumetric representation. The position of the VR controller corresponds to the sagittal, axial and coronal images, which are dynamically displayed on the sides of boxes containing the patient's representations. VR, virtual reality.

of the transfer function allowed efficient visualisation of the tumour boundaries. Here, the ability to grasp and freely orient the patient's avatar and importantly to cut through the volume was instrumental in providing geometric insights of the tumour within its environment (see video 1). The combined effects of immersion with an accurate to-scale volumetric representation of the patient while being able to literally cut through the data provided an enhanced capacity to visualise the tumour within its local and global environment. The lesions were determined to be strictly in the lower quadrants, as seen in figure 2, with sufficient residual volume for a conservative treatment.

Informed consent was obtained from the patient to undergo a partial mastectomy of the superior pedicle mammoplasty. The patient was aware of the benefits and risks of this procedure, particularly the risk of secondary revision in the case of unhealthy margins and risk of aesthetic sequelae. Preoperatively, we placed two wires in the breast: one in the residual lesion detected by ultrasonography, and another along the coil initially placed, which showed the upper limit of the excision zone.

Additionally, the DIVA software was again used to visualise the lesions in the immediate preoperative period in order to allow the surgeon to precisely determine the resection area (see video 1$)$.

\section{TREATMENT}

Under general anaesthesia, partial mastectomy and superior pedicle mammoplasty were performed guided by the preoperative VR visualisation using the DIVA software platform. We were able to excise the breast tissue that included the cancerous lesions detected prior and after chemotherapy with adequate safety margin. Such limited excision allowed a successful superior pedicle mammoplasty. There were no intraoperative complications. Recovery from anaesthesia was uneventful.

\section{OUTCOME AND FOLLOW-UP}

The patient did not present any postoperative complications and was discharged from the hospital the same day of the surgery. On final pathological examination of the excised lesions, there 


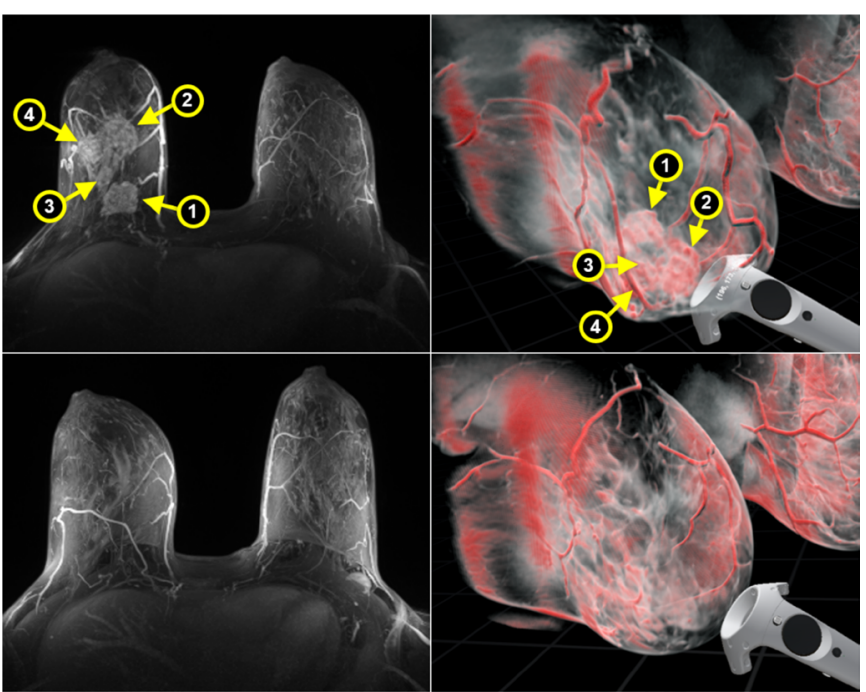

Figure 2 Visualisation of T1-weighted contrast-enhanced MRI prechemotherapy and postchemotherapy. (Top left) Maximum intensity projection of axial MRI taken prior to chemotherapy. Four multifocal lower quadrant lesions are observed in the right breast; (1) corresponds to a single bilobed mass in the lower inner quadrant and (2), (3) and (4) correspond to three contiguous masses at the union of the lower quadrants. (Top right) The same MRI is visualised using the DIVA software in virtual reality revealing the three-dimensional extent and localisation of the lesions in the lower breast identified on MRI. (Bottom left) Maximum intensity projection of axial MRI taken after chemotherapy. Previous lesions are greatly diminished. (Bottom right) The DIVA visualisation of the same MRI indicates the missing lesions due to treatment response. Note that figures extracted from DIVA cannot reproduce the stereoscopic volumetric effect experienced by a user wearing the virtual reality headset.

was mammary parenchyma with fibrous scarring, indicating a complete response to neoadjuvant treatment (residual cancer burden of 0 ). Figure 1 shows the macroscopic and histopathology images of the surgical specimen at one of the identified lesions, indicating a fibrous reorganisation associated with a polymorphic inflammatory infiltrate linked to the chemotherapy treatment. Thirteen axillary lymph nodes were removed and were free of tumour invasion, without fibrous scarring (International Union Against Cancer Tumor-Nodes-Metastasis stage version 8: ypT0N0). Figure 3 displays the photos of the patient prior to and after breast-conserving surgery.

\section{DISCUSSION}

The treatment of unifocal breast cancer lesions is well recognised and may, in the case of sufficient breast volume, include a partial mastectomy followed by radiotherapy.

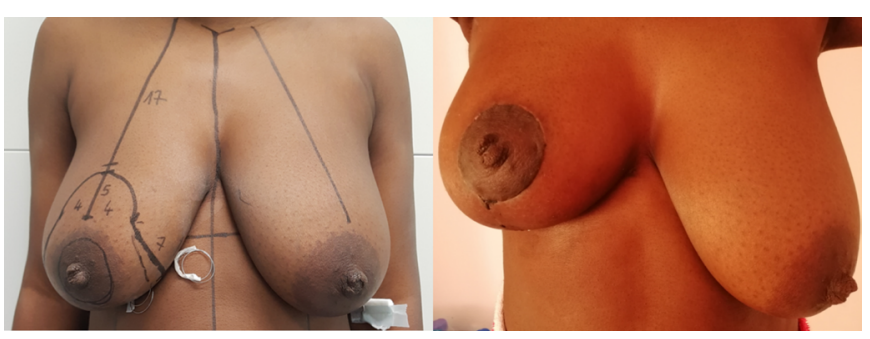

Figure 3 Photos of the patient prior to and after breast-conserving surgical treatment.
In the case of multiple breast cancer lesions, MF lesions are differentiated from multicentric (MC) lesions. MF cancers have been defined as more than one cancer within the same breast quadrant, whereas MC cancers are widely spaced in different quadrants. The incidence of multiple breast cancer lesions ranges from $20 \%$ to $25 \%$, and has likely increased in recent years with the increased use of MRI. ${ }^{4}$

For MC lesions, the currently recommended treatment is radical mastectomy, mainly based on retrospective series showing an increase in recurrence rate with conservative treatment. ${ }^{5-7}$

More recently, studies have shown the feasibility and safety of conservative treatment on this type of lesion, particularly with oncoplastic surgery. ${ }^{8-12}$

NAC, that is, chemotherapy initiated before breast cancer surgery, was initially used to permit surgery in inoperable locally advanced breast cancers. It was later extended to patients who have operable breast cancer but not manageable by breastconserving surgery to preserve the breast. NAC in such patient populations allowed breast-conserving surgery. No difference in survival has been shown between patients managed with adjuvant and neoadjuvant strategies. ${ }^{13}$ However, the neoadjuvant strategy can increase the prospect of breast-conserving surgeries and demonstrate the efficacy of a particular chemotherapy regimen. ${ }^{13}$

Since the CREATE-X study, NAC has become the first-line therapy in triple-negative tumours, with the possibility of postneoadjuvant treatment with capecitabine, in case of partial response to NAC, with a survival benefit. ${ }^{14}$

In this patient, NAC was considered more with regard to the possibility of postneoadjuvant treatment, rather than obtaining a conservative treatment, as the initial lesion was extensive and would likely require a radical mastectomy. However, given the good clinical and radiological response, and the breast volume, the question of a conservative treatment with breast-conserving surgery was raised.

Visualisation of the patient's MRI by common radiological two-dimensional visualisation in complex cases with multiple lesions does not allow a good understanding of actual breast volumes and the precise localisation of lesions for many surgeons. The patient's avatars generated with the DIVA software provided access to a full volumetric understanding of the tumour position within the breast and 3D geometries in relation to the mammary tissue and allowed, through the interactive VR cutting tools, navigation within the tumour to better understand its boundaries. The generation of patient representation from volumetric ray casting does not require tissular boundaries to be predefined. Hence, when handling tumours with ill-defined boundaries, such is often the case with a breast MRI, the VR representation allows boundary regions to be analysed in an unbiased fashion. We performed various image captures within the virtual interface to use for guidance, as shown in figure 2.

In this case, VR visualisation of the MRI with DIVA allowed us to confirm that the ratio between the excision volume and the residency volume would allow for oncoplastic surgery to be performed. Furthermore, the multidisciplinary use of DIVA between the surgeon (ie, for visualisation and preparation of the surgery) and the radiologist (ie, for image preparation and discussion of results) led to a clear treatment consensus.

Within this application, the main limitation of DIVA is that the position of the avatar corresponds to the position seen on MRI (breast down in the prone position) and not the surgical supine position. Hence, expected mechanical modifications of the breast due to different positions were not modelled within the patient's avatar. Yet it was still possible to move the avatar 
in the surgical supine position to gain a good impression of the cancerous lesion configuration prior to the operation.

In conclusion, the use of VR, here DIVA, as a surgery planning tool can allow more conservation of breast tissue during the surgical excision of malignant tumours. This increases the odds of implementing oncoplastic surgery with less disfigurement and without compromising patient safety.

\section{Learning points}

- Virtual reality-based visualisation of the native MRI grants surgeons an enhanced understanding of tumour localisation and breast volumes.

- Virtual reality visualisation of tumour and breast volumes can increase the incidence of breast-conserving surgeries allowing successful oncoplastic procedures.

- Virtual reality visualisation of breast lesions with the DIVA software platform can enhance the safety of breastconserving surgery.

- The DIVA software platform is a user-friendly means to immerse the breast surgeon in the anatomical pathology of the breast lesion.

\section{Twitter Mohamed El Beheiry @MoElbeheiry}

Acknowledgements We thank Professor Fabien Reyal (Institut Curie) for his support in the preparation of this manuscript. We thank Guillaume Bataillon (Institut Curie) for his macroscopic and histopathological analyses of the initial biopsy and surgical specimen of the patient.

Contributors EL, J-BM and MEB wrote the manuscript. MEB and J-BM developed the DIVA software. EL performed the surgery. CM prepared and analysed the images.

Funding Roche France provided financial support for the construction of the Institut Curie neoadjuvant database (NEOREP).

Disclaimer The funding source had no role in data analysis and interpretation or in the writing of the manuscript.

Competing interests MEB and J-BM are cofounders and shareholders and, respectively, Chief Technology Officer and Chief Scientific Officer of AVATAR MEDICAL SAS, a startup that commercialises software for surgery planning in virtual reality. The DIVA software used in this study is not being commercialised by AVATAR MEDICAL SAS but was based on the same technology. The DIVA software used in this study is freely available and is reported in El Beheiry M, et al, 'DIVA: Natural Navigation Inside 3D Images Using Virtual Reality', J Mol Biol. 432(16): 4745-9 (2020).
Patient consent for publication Obtained.

Provenance and peer review Not commissioned; externally peer reviewed.

Open access This is an open access article distributed in accordance with the Creative Commons Attribution Non Commercial (CC BY-NC 4.0) license, which permits others to distribute, remix, adapt, build upon this work non-commercially, and license their derivative works on different terms, provided the original work is properly cited and the use is non-commercial. See: http://creativecommons.org/ licenses/by-nc/4.0/.

\section{ORCID iDs}

Enora Laas http://orcid.org/0000-0003-3875-4011

Mohamed El Beheiry http://orcid.org/0000-0001-9827-6619

Jean-Baptiste Masson http://orcid.org/0000-0002-5484-9056

Caroline Malhaire http://orcid.org/0000-0002-9235-9816

\section{REFERENCES}

1 El Beheiry M, Godard C, Caporal C, et al. DIVA: natural navigation inside 3D images using virtual reality. J Mol Biol 2020;432:4745-9.

2 Bouaoud J, El Beheiry M, Jablon E, et al. DIVA, a 3D virtual reality platform, improves undergraduate craniofacial trauma education. J Stomatol Oral Maxillofac Surg 2020;431. doi:10.1016/j.jormas.2020.09.009. [Epub ahead of print: 29 Sep 2020].

3 Engel K, Hadwiger M, Kniss J R-S. Real-Time volume graphics. 1st edn. A K Peters/CRC Press., 2006.

4 Winters ZE, Horsnell J, Elvers KT, et al. Systematic review of the impact of breastconserving surgery on cancer outcomes of multiple ipsilateral breast cancers. BJS Open 2018;2:162-74

5 Wilson LD, Beinfield M, McKhann CF, et al. Conservative surgery and radiation in the treatment of synchronous ipsilateral breast cancers. Cancer 1993;72:137-42.

6 Kurtz JM, Jacquemier J, Amalric R, et al. Breast-Conserving therapy for macroscopically multiple cancers. Ann Surg 1990;212:38-44.

7 Leopold KA, Recht A, Schnitt SJ, et al. Results of conservative surgery and radiation therapy for multiple synchronous cancers of one breast. Int I Radiat Oncol Biol Phys 1989;16:11-16.

8 Nijenhuis MV, Rutgers EJT. Conservative surgery for multifocal/multicentric breast cancer. Breast 2015;24 Suppl 2:S96-9.

9 Kadioğlu H, Yücel S, Yildiz S, Yildiz Şeyma, et al. Feasibility of breast conserving surgery in multifocal breast cancers. Am J Surg 2014;208:457-64.

10 Ninkovic S, Ninkovic V, Cvetkovic A, et al. Multifocal and multicentric breast cancer: is breast conserving surgery acceptable? J Buon 2012;17:38-45.

11 Bauman L, Barth RJ, Rosenkranz KM. Breast conservation in women with multifocalmulticentric breast cancer: is it feasible? Ann Surg Oncol 2010;17(Suppl 3):325-9.

12 Lim W, Park E-H, Choi S-L, et al. Breast conserving surgery for multifocal breast cancer Ann Surg 2009;249:87-90.

13 Early Breast Cancer Trialists' Collaborative Group (EBCTCG). Long-Term outcomes for neoadjuvant versus adjuvant chemotherapy in early breast cancer: meta-analysis of individual patient data from ten randomised trials. Lancet Oncol 2018;19:27-39.

14 Masuda N, Lee S-J, Ohtani S, et al. Adjuvant capecitabine for breast cancer after preoperative chemotherapy. N Engl J Med 2017;376:2147-59.

Copyright 2021 BMJ Publishing Group. All rights reserved. For permission to reuse any of this content visit

https://www.bmj.com/company/products-services/rights-and-licensing/permissions/

BMJ Case Report Fellows may re-use this article for personal use and teaching without any further permission.

Become a Fellow of BMJ Case Reports today and you can:

- Submit as many cases as you like

- Enjoy fast sympathetic peer review and rapid publication of accepted articles

- Access all the published articles

- Re-use any of the published material for personal use and teaching without further permission

Customer Service

If you have any further queries about your subscription, please contact our customer services team on +44 (0) 2071111105 or via email at support@bmj.com.

Visit casereports.bmj.com for more articles like this and to become a Fellow 